\title{
Function-Oriented Measurements of Micro Gears for Lifetime Evaluation
}

\author{
Benjamin Haefner ${ }^{1}$, Gisela Lanza ${ }^{1}$ \\ ${ }^{1}$ wbk Institute of Production Science, Karlsruhe Institute of Technology (KIT), \\ Kaiserstraße 12, 76131 Karlsruhe \\ benjamin.haefner@kit.edu
}

\begin{abstract}
:
Nowadays, micro transmissions are used in combination with micro motors in manifold industrial applications, e.g. in the medical industry in actively controlled prostheses. Micro transmissions consist of micro gears with a module $<200 \mu \mathrm{m}$. The main function of micro gears is a proper operation over the required lifetime. Lifetime evaluation is particularly important for micro gears, as the influence of geometric shape deviations is very large. Efficient production metrology is based on function-oriented measurements. Thus, in this article, a methodology is introduced to enable a function-oriented evaluation of micro gear measurements. For this purpose, high-precision 3D CMM measuring data are processed by finite element method (FEM) simulation to calculate the characteristic loads. These are correlated with experimental data of lifetime experiments.
\end{abstract}

Key words: Function-oriented metrology, micro gears, lifetime, CMM, finite element method

\section{Introduction}

Micro transmissions are important micro systems which are today used in combination with micro motors in manifold industrial applications. In medical engineering, for instance, they are applied for dental drills, equipment for minimal-invasive surgery or actively controlled prostheses. In industrial automation, hexapod systems with micro transmissions are used for wafer processing. Micro gears, which are defined as gears with a module < $200 \mu \mathrm{m}$ [1], are fundamental components in micro transmissions. In most applications of micro transmissions they are manufactured from steel as spur gears by hobbing. Because of the small dimensions of the gears and the technological limits of the available machines, they show significantly larger shape deviations in relation to their part size than conventional gears. This can lead to a malfunction of the rolling motion of the transmission, which causes a shortening of its lifetime.

Because of this, a precise understanding of the influence of the geometrical deviations on the expectable lifetime is particularly crucial in the case of micro gears. For macro gears in most cases this relationship can be described adequately by the ISO standard 6336 [2], by which the load capacity is approximated dependent on certain tolerances, e.g. defined in
DIN 3962 [3]. The standards for tolerance classification such as DIN 3962, however, only refer to gears with a module $>1 \mathrm{~mm}$. Furthermore, Braykoff experimentally demonstrated that calculating the load capacity of micro gears according to ISO 6336 is very imprecise [4]. Hence, currently, there is a lack of knowledge how the relatively large geometrical deviations of micro gears impair their functional rolling motion over the operating time.

Thus, the research approach of this article deals with the development of a model which represents the influence of measured geometrical deviations on the lifetime of micro gears. By means of this model a more accurate lifetime prognosis will be possible. From this, inferences regarding the optimal design of the gear tolerances can be drawn. A required decrease of the tolerances leads to longer lifetime, while an expansion of the tolerance range of noncritical characteristics can reduce the production costs.

From a metrological viewpoint, the research approach deals with the development of a function-oriented evaluation method for micro gears. Function-oriented metrology, has already been investigated e.g. for the evaluation of micro-structured surfaces [5]. Considering the lifetime of micro gears, this research approach, broadens the scope of function-oriented 
measurements with regard to a temporal dimension.

In section 2 of this article an overview of the research approach is shown, before the main steps are elaborated in sections 3 to 6 . Section 7 concludes with an outlook on ongoing research.

\section{Function-Oriented Metrology of Micro Gears for Lifetime Evaluation}

In order to draw reliable conclusions of the influence of the measured gear geometry on the lifetime of micro gears, an adequate model of their relationship is necessary. The generation of the model is realized by a fourstep methodology, which is illustrated in Fig. 1 $[6,7]$ :

- Conduction of lifetime experiments

- Measurements of the gear geometry

- Load calculations by means of finite element (FE) simulation

- Determination of lifetime model

First, gear pairs of the respective type are operated in lifetime experiments with clearly defined, realistic conditions, until a defect of one of the gears occurs. The experiments are conducted by means of a high-precision experimental setup. Before the lifetime experiments and at certain times during the experiments the geometry of the gears is measured using a coordinate measuring machine (CMM) with very high accuracy. For both gears, CAD models of the measured gear geometries are generated from the resulting data points. These serve as an input for subsequent finite element simulations, by which the present mechanical loads are calculated at each investigated time of the experiments, considering the real experimental conditions as well as the gear geometries. Based on the loads determined by the FEM calculations and the times of gear defects resulting from the lifetime experiments, a lifetime model dependent on the measured gear geometry can be deduced by applying adequate methods of statistical reliability theory.

In the scope of the current investigations the research approach is focused on the modelling of the rolling action of a gear pair with identical number of teeth. At present, only tooth root break is considered as cause of defect, which significantly is the dominating cause, if micro gears are operated with high torque loads in industrial practice.

For the practical validation of the research approach, currently, gear pairs of hobbed spur gears made from steel are used, which have an involute geometry with $z=17$ teeth, a module of $m=175 \mu \mathrm{m}$ and a width of $b=2 \mathrm{~mm}$. In order to simplify the clamping of the gears in the experimental setup, a gear type with integrated shaft was selected. Within the experiments the gears are operated at a rotational speed of $n=500 / \mathrm{min}$ and a torque of $M=50 \mathrm{mNm}$.

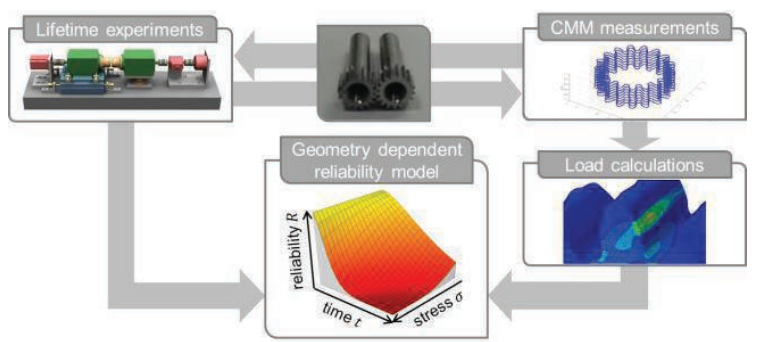

Fig. 1. Methodology for the modelling of the gear lifetime dependent on the measured geometry.

\section{Lifetime Experiments}

For the conduction of the lifetime experiments a high-precision experimental setup is necessary. By means of this, a gear pair can be operated at clearly defined conditions, until a tooth root break occurs. The experimental setup has to comply with the following requirements:

- Accurately defined positioning and fixing of the gears to each other (even after interruptions of the experiments due to geometrical measurements)

- Accurate adjustability of the rotational speed and the torque

- Identification of the time of a gear defect and the broken tooth of the gear pair

- Stability against perturbations of the environment (e.g. temperature, external vibration, contamination etc.)

The requirements of the experimental setup with regard to its geometrical accuracy and its stiffness are very high, as even small inaccuracies of the positioning of the gears lead to a significant impairment of the rolling conditions due to their small size. The experimental setup, which was realized at the Institute of Production Science (wbk), is illustrated in Fig. 2. It was developed according to the systematic approach for designing technical products of VDI 2221.

In the experimental setup defined parameters of the rotational speed and the torque can be set by means of a controllable synchronous motor and hysteresis brake. The rotational speed and the torque are continuously measured with a sensor. 
The rotary motion of the gears is realized by two high-precision spindles. Considering the flanges of the spindles, these have a concentricity of $<2 \mu \mathrm{m}$ and a radial stiffness of $>100 \mathrm{~N} / \mu \mathrm{m}$. At the flange of each spindle a zero-point fixturing system is mounted, by which the micro gears can be clamped on pallets with adjusting elements. One of the two spindles is fixed to an additional zero-point fixturing system. By means of the zero-point fixturing systems the gears can be removed from the experimental setup during a lifetime experiment for intermediate geometric measurements and after those precisely reclamped into the experimental setup. As experimentally verified by the PhysikalischTechnische Bundesanstalt (PTB) the zero-point fixturing systems used have a repetitive accuracy of $<0.1 \mu \mathrm{m}$. The adjusting elements mounted on the zero-point fixturing systems enable a true running accuracy of the clamped gear shafts of $<1 \mu \mathrm{m}$ by means of three adjusting screws in radial direction and six adjusting screws in axial direction.

In order to guarantee an axially parallel positioning of the gear shafts to each other with a defined center distance at the beginning of a series of experiments, one of the two spindles is mounted to a positioning unit with 12 adjusting screws enabling two translational and two rotational degrees of freedom. The resolution of the adjusting screws is $0.5 \mu \mathrm{m}$.

The vibrations of the experimental setup are damped by a massive granite plate, on which all components are mounted. The experimental setup is located in an air-conditioned metrology laboratory, in which a stability of the temperature with deviations $<0.5 \mathrm{~K}$ can be realized.

Within the experimental procedure of the lifetime experiments, first, each examined gear is marked to enable a defined allocation of the interacting teeth. After cleaning by means of an ultrasonic bath, an experimental pair of gears is clamped and precisely oriented by the adjusting elements on the pallets of the zero-point fixturing systems. Then the geometry of both gears is measured, as described in detail in chapter 4.

After this, the pallets are clamped in the experimental setup by first de-clamping the zero-point fixturing system below the spindle. Before starting an experiment, lubrication is applied on the gears and the desired rotational speed and torque are set.

The conduction of each experiment is interrupted in defined intervals for geometrical measurements of the gears. At these times, the pallets are de-clamped from the experimental setup and clamped by a zero-point fixturing system at the micro coordinate measuring machine. Thus, by interrupting a lifetime experiment several times, the change of the geometry of the gears can be sequentially evaluated.

Each experiment is conducted, until a gear defect occurs. For the gears evaluated in the current research project, only tooth root break is considered as cause of defect. The time of defect is determined by an evaluation software which continuously monitors the rotational speed of the gears during the experiments by means of the sensor in the experimental setup. If a tooth root break of one of the gears occurs, a significant decrease of the rotational speed can be observed. The identification of the respective tooth of the tooth root break can be realized by means of an optical microscope after de-clamping the gears from the experimental setup.

Hence, in summary, the results of each lifetime experiment are the determination of the time of the gear defect and the identification of the defect tooth. These data serve as inputs for the determination of the lifetime model.

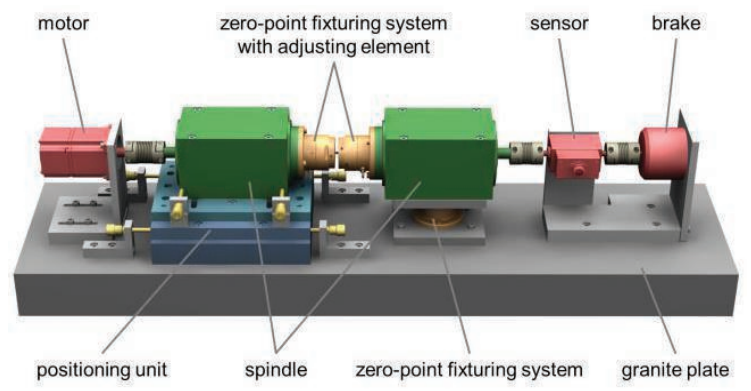

Fig. 2. Schematic representation of the measurement setup for the lifetime evaluation of micro gears.

\section{Measurements of Gear Geometry}

For a function-oriented assessment of the geometry of micro gears both the measurement of data points with very low measurement uncertainty and a meaningful evaluation of the measurement data are very important. The measurements of the gears can be conducted at the Institute of Production Science (wbk) by means of the micro coordinate measuring machine (CMM) Zeiss F25, which is specified by a volumetric length measuring error of $M P E_{E}=(0.25+\mathrm{L} / 666) \mu \mathrm{m}$. At the CMM, the aforementioned zero-point fixturing system is mounted for the fixing of the pallets with the clamped gears, which are also applied in the experimental setup. As the changing accuracy of the zero-point fixturing systems is $0.5 \mu \mathrm{m}$, a reproducible positioning of the gears in the CMM is facilitated. 
In order to enable a function-oriented measurement of the gear geometry for the evaluation of the lifetime, data points are recorded at the tooth roots, tooth flanks and tooth tips in a grid with a point distance of 10 $\mu \mathrm{m}$. For the examined gears the measurements are conducted with a tactile probe with a diameter of $120 \mu \mathrm{m}$. The data points are measured by several circumferences in profile direction at different positions in width direction of the gear (cf. Fig. 3). The measurements are conducted in freeform scanning mode because of the changing gear geometry during the lifetime experiments.

Based on the resulting point cloud of a measurement, which represents the topology of the gear surface with very high accuracy, a CAD model of the measured gear geometry is generated. This can be realized by means of a two-step procedure. First, an areal model based on B-splines is generated, which is then transferred into a volumetric model (cf. Fig. 3).

For the generation of the areal model a twodimensional B-spline surface is constructed from the measured data points. According to [8] the primary B-spline surface is transferred into a surface of Non Uniform Rational B-Splines (NURBS), which is the common B-spline representation of CAD models [9]. Furthermore, NURBS of the end surfaces at the top and at the bottom of the gear are generated and combined with the aforementioned NURBS surface in flank direction. After this, according to [10], the NURBS surface in flank direction is divided into defined sub-surfaces for the tooth flank areas, the tooth root areas and the tooth tip areas to simplify the data processing of the finite element simulation. For the finalization of the areal representation the entire NURBS surface is stored as CAD model in the universal CAD format IGES [11]. The described methodology can be implemented as an automated script by means of the software Mathworks MATLAB.

The second step of the procedure, the transformation of the areal CAD model into a volumetric CAD model, enables the adding of the material characteristics of the gear (e.g. material, density etc.). This can be realized by means of standard CAD software such as Siemens NX 9. The two final volumetric CAD models of a gear pair can be imported into $\mathrm{FE}$ software as inputs for the load simulation of the measured gears.

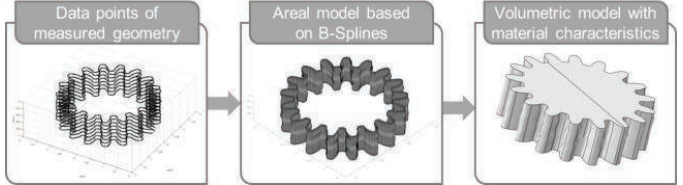

Fig. 3. Procedure for the creation of a CAD model from measured gear data.

\section{Load Calculations by Means of FEM}

The goal of the finite element simulations is to calculate the mechanical loads which occur during the operation of an examined gear pair in the experimental setup. For the focused cause of defect, the tooth root break, the tooth root stress is commonly considered as characteristic property on the basis of the cumulative damage hypothesis of reliability theory [12]. In the finite element simulations of the micro gears, the tooth root stress is evaluated according to the von-Mises-criterion. For each tooth root of both gears, the tooth root stress is determined in different areas in tooth trace direction. For each geometric measurement of an examined gear pair within the lifetime experiments a FE simulation of the respective mechanical loads is conducted. Besides the CAD models of the measured micro gears the experimental conditions of the lifetime experiments in the experimental setup (e.g. position of the gears, rotational speed, and torque) are incorporated in the FE simulations.

The simulation is realized as three-dimensional finite element model in the software Abaqus 6.12. In this model the tooth root stress is evaluated for each teeth interaction. Thus, the gears are rotated in small angles and simulated over the entire turn. In Fig. 5 am exemplary FE simulation of a teeth interaction based on measured geometry data is shown [6]. A detailed description of the FE model can be found in [13].
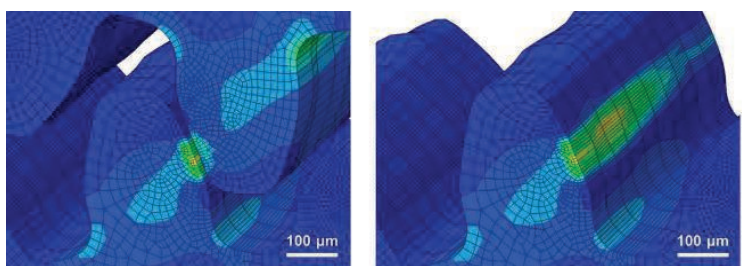

Fig. 4. FE simulation based on measured gear geometries for load calculation [6].

\section{Determination of Lifetime Model}

In order to determine a lifetime model of the micro gears, the goal is to correlate the data of the gear defects of the lifetime experiments with the loads calculated by the FE simulations in an adequate way. 
First, load collectives dependent on the load cycles calculated in the FE simulations are to be determined for each gear pair, assuming the cumulative damage hypothesis [14]. There a several potential methods for this, e.g. various regression models or artificial neural networks [15]. After the conduction of experimental series of the current research, suitable methods for this specific purpose will be evaluated, selected and tested.

Second, the actual lifetime model is developed by means of methods of statistical reliability theory [14]. The inputs of the model, on the one hand, are the times of the gear defects and the identified defect teeth in the lifetime experiments, as well as, on the other hand, the load collectives of the gear pairs for the tooth pairs interacting with each other. As a result of the model the reliability of a gear pair, i.e. its probability of survival, can be modelled dependent on the load collectives, which are determined by the measured geometries of the gears, and the time of gear operation to be evaluated (cf. Fig. 5).

A potential approach for the determination of the model is the method of load-dependent Weibull analysis [16]. Assuming that the interrelationship of the model can be characterized by a two-parametric Weibull distribution, which form parameter $\beta$ is independent of the load and constant, and furthermore assuming that the interrelationship between the present load collective and the damaging of the gear can be modelled by the Inverse Power Law, the following model specification for the reliability $F(t ; \sigma)$ of a gear pair can be deduced:

$F(t ; \sigma)=1-\exp \left(-\left(K \cdot \sigma^{N} \cdot t\right)^{\beta}\right)$

Referring to the load $\sigma$, the gradient of the damaging $\dot{S}$ and the model parameters $K$ and $N$ the Inverse Power Law is defined by [16]:

$\dot{S}=\frac{1}{K \cdot \sigma^{N}}$

The determination of the model parameters can be realized, for instance, by means of maximum-likelihood estimation (MLE) in the context of Weibull analysis.

The detailed elaboration of a suitable method for the determination of the lifetime model is subject to ongoing research at the Institute of Production Science (wbk).

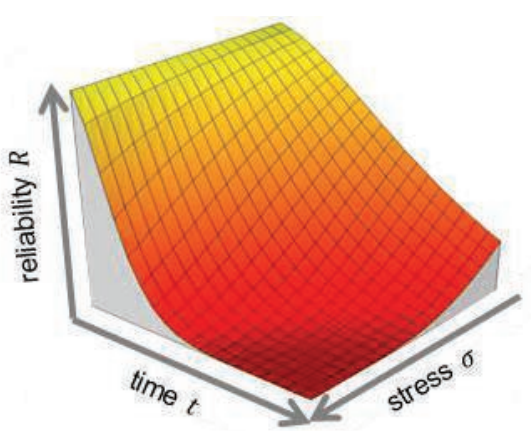

Fig. 5. Schematic illustration of the lifetime model.

\section{Conclusion and Outlook}

Micro gears are important mechanical components which today are used in manifold industrial applications, e.g. in medical engineering. Due to the large geometrical deviations in relation to the part size of micro gears, a significant influence of the manufacturing quality on the functional compliance over the time of operation can be expected. Hence, the object of investigation of this article is the development of a model, which represents the influence of measured geometrical deviations on the lifetime of micro gears. The research approach is structured by a four-step procedure dealing with the conduction of lifetime experiments, geometrical measurements of the micro gears, load calculations by means of FE simulations as well as the final determination of the lifetime model.

Gear pairs are operated in lifetime experiments with defined conditions by means of a highprecision experimental setup, until a tooth root break of a gear occurs. Before and at certain times during the conduction of the lifetime experiments the geometry of the gears is measured by means of a CMM with very low measurement uncertainty and CAD models based on B-splines are generated from the measured data points. By means of those the tooth root stress can be calculated for each measured data set using FE simulations. A reliability model of the gear pairs can be deduced dependent on the exploited load spectra and the failure data from the lifetime experiments. The model can be used for both the evaluation and the prognosis of the functional capability of the micro gear over the desired lifetime. In the continuative research at the Institute of Production Science (wbk) the presented methodology is validated by means of exemplary micro gears, conducting extensive lifetime experiments, geometrical measurements and FE simulations. On the basis of the experimental data the lifetime model is deduced for the case of the exemplary gears. 


\section{References}

[1] Verein Deutscher Ingenieure (ed.), VDI 2731: Microgears, basic principles, Düsseldorf, VDI, 2009

[2] International Organization for Standardization (ed.), ISO 6336-1: Calculation of load capacity of spur and helical gears, Berlin, Beuth, 2006

[3] Deutsches Institut für Normung (ed.), DIN 3962: Tolerances for Cylindrical Gear Teeth, Berlin, Beuth, 1978

[4] C. Braykoff, Tragfähigkeit kleinmoduliger Zahnräder, dissertation, University of Munich, 2007

[5] A. Weckenmann et al., Function-oriented method for the definition and verification of microstructured surfaces, Precision Engineering $37,684-693$ (2013)

[6] G. Lanza et al., Methodology for the lifetime prediction of micro gears dependent on present shape deviations and the material structure, ESREL 23, Amsterdam, 2663-2669 (2013)

[7] B. Haefner et al., Finite Element Analysis of Micro Gears for the Prediction of their Lifetime, euspen 14, Dubrovnik, 277-280 (2014)

[8] D. M. Spink, NURBS Toolbox, http://www. mathworks. com/matlabcentral/fileexchange/ 26390-nurbs-toolbox-by-d-m-spink, 2010, access: March $28^{\text {th }} 2014$

[9] R. Mukundan, Advanced Methods in Computer Graphics, London, Springer, 2012

[10] NN, B-spline Curves: Subdividing a B-spline Curve, http://www.cs.mtu.edu/ shene/ COURSES/cs3621/NOTES/spline/Bspline/ subdivision.html, 1998, access: March $20^{\text {th }} 2014$

[11] NN, igesout, http://www.mathworks.com/ matlabcentral/ fileexchange/14470-igesout, 2009, access: March $14^{\text {th }} 2014$

[12] E. Haibach, Betriebsfestigkeit, Verfahren und Daten zur Bauteilberechnung, $3^{\text {rd }}$ ed., Berlin, Springer, 2006

[13] B. Haefner et al., Finite Element Simulation for Quality Dependent Lifetime Analysis of Micro Gears, CIRP CMMO 15 (accepted), Karlsruhe (2015)

[14] B. Bertsche, Zuverlässigkeit im Fahrzeug- und Maschinenbau, $3^{\text {rd }}$ ed., Berlin, Springer, 2004

[15] K. Siebertz et al., Design of Experiments (DoE), $1^{\text {st }}$ ed., Heidelberg, Springer, 2010

[16] B. W. Nelson, Accelerated Testing, Statistical Models, Test Plans, and Data Analysis, $2^{\text {nd }}$ ed., New York, Wiley, 2004 